\title{
Association between dietary patterns and glycaemic control in a middle-aged Chinese population
}

\author{
Xiao-Ming Shen ${ }^{1}$, Long Shu' ${ }^{2}$, Yi-Qian Huang ${ }^{3}$, Xiao-Yan Zhang ${ }^{2}$, Pei-Fen Zheng ${ }^{2,3}$ and \\ Qin Zhu 2,3,* \\ 'Department of Endocrinology, The No.1 People's Hospital of Pinghu, Pinghu, Zhejiang, The People's Republic of \\ China: ${ }^{2}$ Department of Nutrition, Zhejiang Hospital, Lingyin Road Number 12, Xihu District, Hangzhou 310013 , \\ Zhejiang, The People's Republic of China: ${ }^{3}$ Department of Digestion, Zhejiang Hospital, Lingyin Road Number 12, \\ Xihu District, Hangzhou 310013, Zhejiang, The People's Republic of China
}

Submitted 25 February 2021: Final revision received 16 August 2021: Accepted 6 September 2021: First published online 13 September 2021

\begin{abstract}
Objective: The present work was performed to analyse the association of dietary patterns with glycaemic control (Hb A1c $<7 \%$ ) in a large group of Chinese adults aged between 45 and 59 years.

Design: Habitual dietary intakes in the preceding 12 months were assessed by welltrained interviewers using a validated semi-quantitative FFQ. Factor analysis with principal component method was used to obtain the dietary patterns, and the associations between dietary patterns and glycaemic control were determined using multivariable logistic regression models. Poor glycaemic control was defined as $\mathrm{HbA} 1 \mathrm{c} \geq 7 \cdot 0$.

Setting: Despite decades of research, data on the relationship between dietary patterns and glycaemic control (HbA1c $<7 \%)$ in China are sparse.

Participants: A total of 1739 participants aged 45-59 years from Hangzhou were included in the final analysis.

Results: Three dietary patterns were ascertained and labelled as traditional southern Chinese, Western and grains-vegetables patterns. After controlling of the possible confounders, participants in the highest quartile of Western pattern scores had greater OR for HbA1c $\geq 7.0 \quad(\mathrm{OR}=1.05$; (95\% CI 1.000, 1.095); $P=0.048$ ) than did those in the lowest quartile. Compared with those in the lowest quartile of grains-vegetables pattern, participants in the highest quartile had lower OR for HbA1c $\geq 7 \cdot 0(\mathrm{OR}=0 \cdot 82$; (95\% CI 0.720, 0.949); $P=0 \cdot 038)$. Besides, no significant relationship between the traditional southern Chinese pattern and HbA1c $\geq 7.0$ was observed $(P>0.05)$.

Conclusions: This study indicated that the Western pattern was associated with a higher risk, and the grains-vegetables pattern was associated with a lower risk for $\mathrm{HbA} 1 \mathrm{c} \geq 7 \cdot 0$. Future prospective studies are needed to confirm our findings.
\end{abstract}

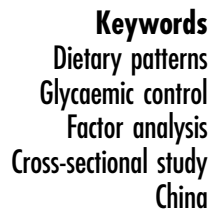

Keywords

Dietary patterns Factor analysis China
Diabetes mellitus (DM) is a global public health issue, with more than 400 million occurring annually worldwide ${ }^{(1)}$. According to the Global Burden of Disease report, DM was recognised as one of the leading causes of years of life lost and has an important effect on Asian countries ${ }^{(2)}$. In China, the overall prevalence of diabetes has increased markedly in the last 10 years, with approximately 114.4 million adults having diabetes and 490 million having prediabetes $^{(3)}$. Obviously, the high prevalence of diabetes carried considerable economic and health burden to the whole Chinese society ${ }^{(4)}$. It is well known that DM is considered as a complex and multifactorial chronic disease that may be influenced by multiple factors, including the family history of DM, genetic factors, sedentary lifestyle, obesity, cigarette smoking and dietary factors ${ }^{(5,6)}$. Therefore, preventing hyperglycaemia and lowering diabetes risk may be effective for the prevention of diabetes in Chinese adults.

Previous studies have suggested that diet and lifestyle modification are modifiable risk factors for the prevention of $\mathrm{DM}^{(7)}$. Traditionally, nutrition research has been focusing on single nutrients or specific foods in isolation. 
Numerous studies have investigated the association between the consumption of individual nutrients or foods and food groups and the risk of $\mathrm{DM}^{(8-15)}$. However, given the complexity of diets and the potential interactions between food components, studying individual nutrients or foods cannot accurately and comprehensively reveal the effect of daily diets on $\mathrm{DM}^{(16)}$. Meanwhile, in daily life, people do not eat isolated nutrients but consume meals containing many combinations of different foods and nutrients $^{(17)}$. In this context, dietary pattern analysis has emerged as an alternative, holistic approach to study the relationship between overall diet and related health consequences, taking into account the complexity of overall diet and potentially facilitate nutritional recommendations ${ }^{(18)}$.

Over the last couple of decades, a rapid nutritional transition characterised by higher intake of sugar and animal-source foods and lower intake of cereals and vegetables is the major contributor to the epidemic of chronic diseases in China ${ }^{(19)}$. Although there is compelling evidence on the associations between dietary patterns and non-communicable diseases, e.g. obesity, hypertension and metabolic syndrome in the Chinese population ${ }^{(20-23)}$, little is known about the role of dietary patterns in glycaemic control $^{(24-26)}$. Heretofore, only one study has investigated the associations between dietary patterns and glycated $\mathrm{Hb}$ A1c (HbA1c) in the Chinese population ${ }^{(26)}$. Also, to our knowledge, no previous studies have examined the impact of empirical dietary patterns on glycaemic control among middle-aged people in China. Against this background, the purpose of this article was to ascertain dietary patterns using factor analysis and subsequently to evaluate the associations between dietary patterns and glycaemic control (HbA1c $<7.0 \%)$ in a middle-aged Chinese population.

\section{Subjects and methods}

\section{Study population}

We used the data set from the Hangzhou Nutrition and Health Survey in China, collected from June 2015 to December 2016. A detailed description of this study has been published previously ${ }^{(16)}$. In brief, 2437 participants aged 45-59 years were recruited when having their annual health examinations at the Medical Center for Physical Examination, 3A Hospital, where participants were interviewed face-to-face by well-trained interviewers using written questionnaire. A flow chart detailing the process of study participants' selection is presented in Fig. 1. Among the eligible participants, we excluded 193 participants with self-reported having history of CVD, cancer or stroke (because of these possible disease-related changes in dietary habits), thirty-six participants with incomplete anthropometric information, 138 participants with the missing or incomplete dietary information in their questionnaires, 275 participants with self-reported having diabetes or taking medications (insulin or oral hypoglycaemic agents), forty-two participants who did not provide the HbA1c measurements and fourteen participants with implausible energy intakes ( $<600$ or $>4000 \mathrm{kcal} / \mathrm{d}$ ). After the mentioned exclusions, in total, 1739 participants (914 men, 825 women) were included in the final analysis. Written informed consent was obtained from all subjects.

\section{Dietary assessment}

We used a semi-quantitative FFQ to assess habitual food intake from the preceding year. This FFQ composing of 138 food items was designed to investigate the dietary intake in the middle-aged Chinese population ${ }^{(16)}$. To evaluate the validity and reliability of this FFQ, a pilot survey in the same population by comparison with three 24-h dietary recalls before the study was performed. The results showed that the correlation coefficients between this FFQ and averages from 24-h dietary recalls ranged between 0.43 and 0.67 for major food groups. In short, these data revealed that this FFQ provided reasonably valid measurements of dietary intake. Participants were asked to recall the frequency of each food item during the previous a year and the estimated portion size, using local weight units (1 Jin $=500 \mathrm{~g}, 1$ Liang $=50 \mathrm{~g}$ ) or natural units (cups). For instance, the frequency response for food consumption included nine categories, as follows: never, $<1$ time a month, 1 to 3 times a month, 1 to 2 times a week, 3 to 4 times a week, 5 to 6 times a week, 1 time a day, 2 times a day and 3 times a day. Standard tableware and food models were provided to help the participants to quantify food consumption accurately. Then, they were converted into an average daily consumption in our analyses. Nutrients intakes (i.e. carbohydrate, protein and fat) based on the food consumption data were computed using the Chinese Food Composition Table ${ }^{(27)}$.

\section{Identification of dietary patterns}

We classified 138 food items from this FFQ into thirty predefined food groups (see online supplemental Table S1) based on culinary usage and common nutrient composition in a middle-aged Chinese population ${ }^{(16)}$. Before beginning the factor analysis, the Bartlett's test of sphericity and Kaiser-Meyer-Olkin (KMO) measure of sample adequacy were used to identify the sample adequacy for factor analysis. Then, we generated dietary patterns by factor analysis (principal components) on the basis of thirty predefined food groups by means of orthogonal transformation (varimax rotation), maintaining the uncorrelated nature of the factors and greater interpretability. The eigenvalue, scree plot and factor interpretability were applied to decide which factors remained ${ }^{(28)}$. After evaluating the eigenvalues, the scree plot test and interpretability, factors with an eigenvalues $\geq 2.0$ were retained. The factor score for each pattern was calculated by summing intakes of food groups weighted by their factor loadings, and each 




Fig. 1 Flow chart of article screening and selection process

participant received a factor score for each identified pattern. In our study, the predefined factor groups with an absolute value factor loading of 0.4 were considered the main factor affecting the pattern. Labelling of dietary patterns was based on the interpretation of foods with high factor loadings for each dietary pattern. Lastly, factor scores were categorised into four equal groups by using quartile cutoffs. Quartile 1 (Q1) represents the lowest adherence, and Quartile 4 (Q4) represents the highest adherence to a certain dietary pattern.

In our previous studies ${ }^{(16,29)}$, three major dietary patterns were extracted and labelled as the traditional southern Chinese'(high consumption of refined grains, vegetables, fruits, pickled vegetables, fish and shrimp, bacon and salted fish, salted and preserved eggs, milk, soya bean and its products, miscellaneous bean, fats and drinks), the 'Western' pattern (high consumption of red meats, poultry and organs, processed and cooked meat, eggs, seafood, cheese, fast foods, snacks, chocolates, alcoholic beverages and coffee) and the 'grains-vegetables' pattern (high consumption of whole grains, tubers, vegetables, mushrooms, vegetable oil, nuts, honey and tea).

\section{Assessment of blood pressure}

The well-trained nurse measured the participant's blood pressure using an automatic device (Omron) with the participants in the sitting position after a 5-10 min rest. Then, three blood pressure readings taken $5 \mathrm{~min}$ apart were recorded, and the mean of the three readings was considered as the final blood pressure.

\section{Assessment of biomarker}

Blood samples were collected from all eligible participants after an overnight fast (at least $8 \mathrm{~h}$ ) and were stored temporarily at $-20^{\circ} \mathrm{C}$ until assessment assays were performed. The 
participants underwent an oral glucose tolerance test using $75 \mathrm{~g}$ of glucose. All blood samples were analysed in the Medical Center for Physical Examination, Zhejiang Hospital for fasting plasma glucose, glycosylated $\mathrm{Hb}$ (HbA1c) using the Hitachi 7180 automatic biochemical analyser (Hitachi, Tokyo, Japan).

\section{Anthropometric measurements}

Weight in light clothes and without shoes was measured with a digital scale to the nearest $0.1 \mathrm{~kg}$, and height was measured without shoes to the nearest $0 \cdot 1 \mathrm{~cm}$. BMI was calculated as weight $(\mathrm{kg})$ divided by the square of height in meters $\left(\mathrm{m}^{2}\right)$. Waist circumference was measured to the nearest $0.1 \mathrm{~cm}$ at the midpoint between the lower rib and the upper iliac crest, while the participants were standing and hip circumstance was measured at the maximum level over light clothing by using an inelastic plastic tape ${ }^{(30)}$. All anthropometric measurements were performed by well-trained nurses according to standard procedures.

\section{Assessment of other variables}

Data on physical activity were collected using the short form of International Physical Activity Questionnaire and was expressed as metabolic equivalent hours per week (MET-h/week) ${ }^{(16)}$. Subsequently, physical activity level was categorised as light, moderate and heavy ${ }^{(31)}$. Additional information including smoking status (never, current and former smokers) and education level (primary school or below, middle and high school, junior college or above) was collected with a written questionnaire. Current smokers were defined as those who smoked at least one cigarette/d. Energy intake was assessed through this semi-quantitative FFQ, and results were expressed in kilocalorie per day $(\mathrm{kcal} / \mathrm{d})$ and categorised according to quartile.

\section{Definition}

Poor glycaemic control was defined as HbA1c $\geq 7 \cdot 0^{(16)}$.

\section{Statistical analyses}

Quartiles were made of three dietary patterns of consumption, and the first quartile (the lowest consumption of the pattern) was compared with the fourth quartile (the highest consumption of the pattern). Data were presented as counts (percentages) for categorical variables and as mean \pm SD for continuous variables. Before data analysis, Shapiro-Wilk test, Q-Q plots and histograms were used to test the normality of distribution. If data are normal distributed variables, we used independent-samples $t$ test to compare the significant differences in continuous variables. Otherwise, the Mann-Whitney test was used. Besides, we used the $\chi^{2}$ test to compare the difference in categorical variables. Multivariate logistic regression analyses were applied to determine the associations of dietary patterns with glycaemic control in crude and adjusted
Table 1 Demographic and clinical characteristics of study participants

\begin{tabular}{|c|c|c|}
\hline Variables & Median ( $n$ 1739) & $\begin{array}{l}\text { Interquartile range } \\
\text { (P25-P75) }\end{array}$ \\
\hline Age (years) & 50 & $47-55$ \\
\hline \multicolumn{3}{|l|}{ Gender, male } \\
\hline$n$ & 914 & \\
\hline$\%$ & 52.6 & \\
\hline BMI $\left(\mathrm{kg} / \mathrm{m}^{2}\right)$ & $24 \cdot 3$ & $22 \cdot 4-26 \cdot 2$ \\
\hline \multicolumn{3}{|l|}{ Obesity } \\
\hline$n$ & 324 & \\
\hline$\%$ & $18 \cdot 6$ & \\
\hline WC $(\mathrm{cm})$ & 84 & $78-90$ \\
\hline \multicolumn{3}{|c|}{ Men: $>85 \mathrm{~cm}$, women $>80 \mathrm{~cm}$} \\
\hline$n$ & 1046 & \\
\hline$\%$ & $60 \cdot 1$ & \\
\hline $\mathrm{HbA1c}$ & $6 \cdot 3$ & $5 \cdot 8-7 \cdot 4$ \\
\hline \multicolumn{3}{|l|}{$>7.0$} \\
\hline$n$ & 970 & \\
\hline$\%$ & $55 \cdot 8$ & \\
\hline SBP (mmHg) & 129 & $118-138$ \\
\hline $\mathrm{DBP}(\mathrm{mmHg})$ & 80 & $71-80$ \\
\hline \multicolumn{3}{|l|}{ Hypertension } \\
\hline$n$ & 498 & \\
\hline$\%$ & 28.6 & \\
\hline \multicolumn{3}{|l|}{ Physical activity } \\
\hline \multicolumn{3}{|l|}{$\begin{array}{l}\text { Light } \\
\text { Litiv }\end{array}$} \\
\hline$n$ & 1376 & \\
\hline$\%$ & $79 \cdot 1$ & \\
\hline \multicolumn{3}{|l|}{ Moderate } \\
\hline$n$ & 275 & \\
\hline$\%$ & $15 \cdot 8$ & \\
\hline \multicolumn{3}{|l|}{ Heavy } \\
\hline$n$ & 88 & \\
\hline$\%$ & $5 \cdot 1$ & \\
\hline Total energy $(\mathrm{kcal} / \mathrm{d})$ & 1916 & $1856-2163$ \\
\hline
\end{tabular}

models. For the multivariate analysis, the model 1 was adjusted for age (years) and sex (male/female); model 2 was further adjusted for educational level (primary school or below, middle and high school, junior college or above), smoking (never, current, former), physical activity (light, moderate and heavy) and BMI (continuous); model 3 was additionally adjusted for total energy intake (kcal/d). All analyses were performed using IBM Statistical Package SPSS (version 23.0, SPSS Inc.), and a 2-sided $P<0.05$ was set as the threshold for statistical significance.

\section{Results}

The demographic and clinical characteristics of study participants are shown in Table 1 . This present sample was composed mainly of male $(52.6 \%)$, with a mean age of 50 years (interquartile range, 47-55). Of the 1739 eligible participants, $18.6 \%$ were obesity, $60.1 \%$ had abnormal waist circumference, $55.8 \%$ lacked glycaemic control (HbA1c $>7 \%$ ) and $28.6 \%$ had hypertension. Total energy consumption was $1916 \mathrm{kcal} / \mathrm{d}$ (interquartile range: 
Table 2 The characteristics of study participants across quartiles of the main dietary pattern scores

\begin{tabular}{|c|c|c|c|c|c|c|c|c|c|c|c|c|c|c|c|}
\hline & \multicolumn{4}{|c|}{$\begin{array}{l}\text { Traditional southern } \\
\text { Chinese pattern score }\end{array}$} & \multirow[b]{3}{*}{$P$} & \multicolumn{4}{|c|}{ Western pattern score } & \multirow[b]{3}{*}{$P$} & \multicolumn{4}{|c|}{$\begin{array}{l}\text { Grains-vegetables } \\
\text { pattern score }\end{array}$} & \multirow[b]{3}{*}{$P$} \\
\hline & \multicolumn{2}{|c|}{$\begin{array}{c}\mathrm{Q} 1 \\
(n 434)\end{array}$} & \multicolumn{2}{|c|}{$\begin{array}{c}\mathrm{Q} 4 \\
(n 435) \\
\end{array}$} & & \multicolumn{2}{|c|}{$\begin{array}{c}\mathrm{Q} 1 \\
(n \text { 435) }\end{array}$} & \multicolumn{2}{|c|}{$\begin{array}{c}\mathrm{Q} 4 \\
(n 435)\end{array}$} & & \multicolumn{2}{|c|}{$\begin{array}{c}\text { Q1 } \\
(n \text { 435) }\end{array}$} & \multicolumn{2}{|c|}{$\begin{array}{c}\text { Q4 } \\
(n 434)\end{array}$} & \\
\hline & $n$ & $\%$ & $n$ & $\%$ & & $n$ & $\%$ & $n$ & $\%$ & & $n$ & $\%$ & $n$ & $\%$ & \\
\hline \multicolumn{16}{|l|}{ Age (years) } \\
\hline Mean & \multicolumn{2}{|c|}{$51 \cdot 3$} & \multirow{2}{*}{\multicolumn{2}{|c|}{$\begin{array}{r}50 \cdot 6 \\
4.7\end{array}$}} & \multirow[t]{2}{*}{0.355} & \multirow{2}{*}{\multicolumn{2}{|c|}{$\begin{array}{r}51.6 \\
4.7\end{array}$}} & \multirow{2}{*}{\multicolumn{2}{|c|}{$\begin{array}{r}50 \cdot 3 \\
4 \cdot 3\end{array}$}} & \multirow[t]{2}{*}{$<0.05$} & \multirow{2}{*}{\multicolumn{2}{|c|}{$\begin{array}{r}50 \cdot 4 \\
4.6\end{array}$}} & $51 \cdot \varepsilon$ & & $<0.05$ \\
\hline SD & 4.5 & & & & & & & & & & & & 4.7 & & \\
\hline BMI $\left(\mathrm{kg} / \mathrm{m}^{2}\right)$ & & & & & & & & & & & & & & & \\
\hline Mean & $24 \cdot 4$ & & $24 \cdot \varepsilon$ & & 0.108 & $24 \cdot 2$ & & $25 \cdot($ & & $<0.05$ & 24.9 & & $24 \cdot($ & & $<0.05$ \\
\hline SD & 2.9 & & $3 \cdot($ & & & $2 \cdot 6$ & & $2 \cdot 9$ & & & $2 \cdot 7$ & & $2 \cdot 6$ & 64 & \\
\hline WC $(\mathrm{cm})$ & & & & & & & & & & & & & & & \\
\hline Mean & 84.3 & & 85 & & 0.120 & 84.2 & & 87. & & $<0.01$ & $86 \cdot 9$ & & $82 \cdot \varepsilon$ & & $<0.01$ \\
\hline SD & $9 \cdot 0$ & & 9. & & & 8.7 & & $8 \cdot($ & & & $9 \cdot 0$ & & $9 \cdot($ & 00 & \\
\hline WHR & & & & & & & & & & & & & & & \\
\hline Mean & $0 . \varepsilon$ & & $0 \cdot \varepsilon$ & & 0.602 & 087 & & $0 \cdot \varepsilon$ & & $<0.05$ & 0.8 & 88 & $0 \cdot \varepsilon$ & 86 & $<0.05$ \\
\hline SD & 0.0 & & $0 \cdot($ & & & 0.0 & & $0 .($ & & & 0.0 & 06 & $0 \cdot($ & 08 & \\
\hline $\mathrm{FPG}(\mathrm{mmol} / \mathrm{l})$ & & & & & & & & & & & & & & & \\
\hline Mean & $5 \cdot 2$ & & $5 \cdot 3$ & & 0.080 & $5 \cdot 1$ & & $5 \cdot \varepsilon$ & 34 & $<0.01$ & $5 \cdot 7$ & 77 & $5 \cdot$ & 35 & $<0.01$ \\
\hline SD & 0.6 & 33 & 0.7 & 71 & & 0.7 & & 0.7 & 73 & & 0.8 & 81 & 0. & 70 & \\
\hline Obesity & 71 & $16 \cdot 4$ & 62 & $14 \cdot 3$ & 0.388 & 50 & 11.5 & 80 & 18.4 & $<0.01$ & 72 & $16 \cdot 6$ & 47 & $10 \cdot 8$ & $<0.001$ \\
\hline Hypertension & 125 & $28 \cdot 8$ & 153 & $35 \cdot 2$ & $<0.05$ & 102 & 23.4 & 142 & $32 \cdot 6$ & $<0.01$ & 130 & 29.9 & 106 & 24.4 & 0.070 \\
\hline Diabetes & 87 & $20 \cdot 0$ & 94 & 21.6 & 0.570 & 72 & $16 \cdot 6$ & 104 & $23 \cdot 9$ & $<0.01$ & 92 & $21 \cdot 1$ & 65 & $15 \cdot 0$ & $<0.05$ \\
\hline Gender & & & & & $<0.001$ & & & & & $<0.05$ & & & & & $<0.001$ \\
\hline Female & 136 & $31 \cdot 3$ & 215 & $49 \cdot 4$ & & 199 & $45 \cdot 7$ & 166 & $38 \cdot 2$ & & 174 & $40 \cdot 0$ & 251 & $57 \cdot 8$ & \\
\hline Male & 298 & $68 \cdot 7$ & 220 & $50 \cdot 6$ & & 236 & $54 \cdot 3$ & 269 & $61 \cdot 8$ & & 261 & 60.0 & 183 & $42 \cdot 2$ & \\
\hline Smoking status & & & & & 0.001 & & & & & $<0.01$ & & & & & $<0.01$ \\
\hline Never & 353 & $81 \cdot 3$ & 307 & $70 \cdot 6$ & & 296 & $68 \cdot 1$ & 268 & $61 \cdot 6$ & & 332 & $76 \cdot 3$ & 366 & $84 \cdot 3$ & \\
\hline Current & 66 & $15 \cdot 2$ & 107 & $24 \cdot 6$ & & 94 & 21.6 & 134 & $30 \cdot 8$ & & 76 & $17 \cdot 5$ & 44 & $10 \cdot 2$ & \\
\hline Former & 15 & 3.5 & 21 & 4.8 & & 45 & $10 \cdot 3$ & 33 & 7.6 & & 27 & $6 \cdot 2$ & 24 & 5.5 & \\
\hline Education & & & & & 0.395 & & & & & $<0.05$ & & & & & 0.268 \\
\hline Primary school or below & 151 & $34 \cdot 8$ & 165 & $37 \cdot 9$ & & 137 & 31.5 & 156 & $35 \cdot 9$ & & 166 & $38 \cdot 2$ & 164 & $37 \cdot 8$ & \\
\hline Middle and high school & 237 & $54 \cdot 6$ & 234 & 53.8 & & 267 & 61.4 & 230 & $52 \cdot 9$ & & 224 & 51.5 & 238 & $54 \cdot 8$ & \\
\hline Junior college or above & 46 & $10 \cdot 6$ & 36 & 8.3 & & 31 & $7 \cdot 1$ & 49 & 11.3 & & 45 & $10 \cdot 3$ & 32 & 7.4 & \\
\hline The average monthly income/person & & & & & 0.167 & & & & & 0.093 & & & & & $<0.05$ \\
\hline$<2000(\mathrm{RMB})$ & 183 & $42 \cdot 2$ & 203 & $46 \cdot 7$ & & 194 & 44.6 & 177 & $40 \cdot 7$ & & 182 & 41.8 & 211 & $48 \cdot 7$ & \\
\hline 2000-4000 (RMB) & 205 & $47 \cdot 2$ & 178 & 40.9 & & 190 & 43.7 & 185 & 42.5 & & 184 & $42 \cdot 3$ & 176 & 40.5 & \\
\hline$>4000(\mathrm{RMB})$ & 46 & $10 \cdot \overline{6}$ & 54 & $12 \cdot 4$ & & 51 & 11.7 & 73 & $16 \cdot 8$ & & 69 & $15 \cdot 9$ & 47 & $10 \cdot 8$ & \\
\hline Physical activity & & & & & 0.422 & & & & & 0.193 & & & & & 0.096 \\
\hline Light & 338 & 77.9 & 348 & $80 \cdot 0$ & & 340 & $78 \cdot 2$ & 357 & $82 \cdot 1$ & & 339 & $77 \cdot 9$ & 333 & $76 \cdot 7$ & \\
\hline Moderate & 78 & $18 \cdot 0$ & 65 & 14.9 & & 62 & $14 \cdot 3$ & 57 & $13 \cdot 1$ & & 79 & $18 \cdot 2$ & 70 & $16 \cdot 1$ & \\
\hline Heavy & 18 & $4 \cdot 1$ & 22 & $5 \cdot 1$ & & 33 & 7.6 & 21 & 4.8 & & 17 & 3.9 & 31 & $7 \cdot 2$ & \\
\hline Total energy $(\mathrm{kcal} / \mathrm{d})$ & & & & & & & & & & & & & & & \\
\hline Mean & $2163 \cdot 7$ & & $1916 \cdot 5$ & & $<0.01$ & $1930 \cdot 8$ & & $2286 \cdot$ & & $<0.001$ & $2122 \cdot 5$ & & $1902 \cdot$ & & $<0.01$ \\
\hline SD & $149 \cdot 6$ & & 162 & & & $160 \cdot 7$ & & 173 & & & $156 \cdot 8$ & & 180 & & \\
\hline
\end{tabular}

WC, waist circumference; WHR, waist:hip ratio; FPG, fasting plasma glucose.

$P$ values for continuous variables (ANOVA) and for categorical variables $\left(\chi^{2}\right.$ test).

Categorical variables are presented as sum and percentages, and continuous variables are presented as mean \pm SD.

1856-2163). The Bartlett's test $(P<0 \cdot 0001)$ and KMO index $(0.798)$ indicated that the correlation between variables was sufficiently robust to perform factor analysis 21. Herein, three main dietary patterns were identified, naming the traditional southern Chinese, western and grainsvegetables patterns, which accounted for $10 \cdot 3 \%, 8.5 \%$ and $6.8 \%$ of the dietary intake variance, respectively. The factor-loading matrixes for these dietary patterns are shown in Supplemental Table S2.

The characteristics of the study participants across quartile categories of the main dietary pattern scores are shown in Table 2. Compared with the study participants in the lowest quartile, those in the highest quartile of the traditional southern Chinese pattern were females, smokers and had higher prevalence of hypertension, lower total energy intake. In contrast, participants who belonged to the highest quartile of the Western pattern were more likely to be younger, males, smokers, with higher BMI, waist circumference, WHR, FBG, educational level, total energy intake and higher prevalence of obesity, hypertension and diabetes than those in the lowest quartile. Besides, in comparison with the participants from the lowest quartile of the grains-vegetables pattern, those in the highest quartile were more likely to be older, females, never-smokers and had lower BMI, waist circumference, WHR, FBG, income and total energy intake and had lower prevalence of obesity, hypertension and diabetes $(P<0.05)$. 
Table 3 Multivariable adjusted $\mathrm{OR}$ and $95 \% \mathrm{Cl}$ for glycaemic control $(\mathrm{HbA} 1 \mathrm{c}<7.0 \%)$ across the quartile $(\mathrm{Q})$ categories of dietary pattern scores in Zhejiang Province, China

\begin{tabular}{|c|c|c|c|c|c|c|c|c|c|c|c|c|}
\hline & \multicolumn{4}{|c|}{$\begin{array}{l}\text { Traditional southern Chinese pattern } \\
\text { score }\end{array}$} & \multicolumn{4}{|c|}{ Western pattern score } & \multicolumn{4}{|c|}{ Grains-vegetables pattern score } \\
\hline & \multirow[b]{2}{*}{ Q1 } & \multicolumn{2}{|r|}{ Q4 } & \multirow[b]{2}{*}{$P$} & \multirow[b]{2}{*}{ Q1 } & \multicolumn{2}{|r|}{ Q4 } & \multirow[b]{2}{*}{$P$} & \multirow[b]{2}{*}{ Q1 } & \multicolumn{2}{|r|}{ Q4 } & \multirow[b]{2}{*}{$P$} \\
\hline & & OR & $95 \% \mathrm{Cl}$ & & & OR & $95 \% \mathrm{Cl}$ & & & OR & $95 \% \mathrm{Cl}$ & \\
\hline Model 1 & 1.00 & $1 \cdot 15$ & $1.014,1.309$ & 0.030 & 1.00 & $1 \cdot 70$ & $1 \cdot 323,2 \cdot 174$ & 0.000 & 1.00 & 0.51 & $0.320,0.817$ & 0.000 \\
\hline Model 2 & 1.00 & 1.09 & $0.902,1.323$ & 0.365 & 1.00 & 1.39 & $1.069,1.842$ & 0.017 & 1.00 & 0.68 & $0.522,0.899$ & 0.009 \\
\hline Model 3 & 1.00 & 1.08 & $0 \cdot 889,1 \cdot 322$ & 0.425 & 1.00 & 1.05 & $1.000,1.095$ & 0.048 & 1.00 & 0.82 & $0.720,0.947$ & 0.038 \\
\hline
\end{tabular}

Model 1: adjusted for sex and age; model 2: further adjusted for educational level (< high school, high school and $>$ high school), physical activity (MET-h/week), smoking status (never, current and former), hypertension (yes/no) and BMl; model 3: additionally adjusted for total energy intake.

Q4: the highest quartile of dietary patterns, Q1: the lowest quartile of dietary patterns (reference).

The association between dietary patterns and glycaemic control by multivariate logistic regression analysis is shown in Table 3. After adjusting for potential confounding variables, study participants in the fourth quartile (Q4, highest consumption) of the Western pattern scores had greater OR for glycaemic control $(\mathrm{OR}=1.05$; $(95 \%$ CI 1.000 , 1.095); $P=0.048)$ than did those in the first quartile (Q1, lowest consumption). Compared with those in the first quartile, participants in the fourth quartile of the grains-vegetables pattern scores had a lower OR for glycaemic control ( $\mathrm{OR}=0.82 ;(95 \%$ CI 0.720, 0.947); $P=0.038)$. Besides, no significant association was established between the traditional southern Chinese pattern and glycaemic control $(P>0.05)$.

\section{Discussion}

In the past three decades, T2DM and its complications have reached epidemic levels, particularly in China. However, reports in the literature on the association of dietary patterns with glycaemic control are scarce. To the authors' knowledge, this is one of the few studies to identify the association of dietary patterns with glycaemic control in a middle-aged Chinese population. In the present study, three main dietary patterns were identified by factor analysis and named as 'traditional Chinese', 'Western' and 'grains-vegetables', respectively. Results from this study showed that the Western pattern was associated with a greater risk for glycaemic control, and the grains-vegetables pattern was associated with a lower risk for glycaemic control. Moreover, no significant association was observed between traditional Chinese and poor glycaemic control.

Driven by rapid economic growth and nutrition transition, China has experienced an increased burden of nutrition-related diseases, e.g. diabetes and obesity ${ }^{(32)}$. Zhejiang province, located in East China, where people's dietary patterns have also undergone the rapid westernisation that changes the traditional pattern to the western pattern ${ }^{(33)}$. Besides, the specifics of the eating habits differ in China depending on the region and cultural practices. People in Zhejiang tend to have a high amount of sweet food and seafood. Meanwhile, with the rapid development of local online-to-offline food delivery, this dieting trend makes foods more convenient and accessible, influencing the local people's dietary behaviours ${ }^{(32)}$. Compared with the home-made foods, take-outs foods often contain higher oil, salt, unhealthy fat or sugars ${ }^{(34)}$. Thus, studying the associations between dietary patterns and glycaemic control in middle-aged adults from Zhejiang Province can be of value.

In the present study, no significant association was observed for the traditional southern Chinese pattern in relation to improved glycaemic control. This finding is inconsistent with a prior research performed in Brazil, which showed that the healthy eating pattern with a high intake of whole carbohydrates, dairy, white meat, fish, fruits and vegetables was significantly associated with reduced fasting plasma glucose, HbA1c and LDLcholesterol levels in patients with type 2 diabetes ${ }^{(35)}$. There are several possible explanations concerning the apparent null finding for this pattern and glycaemic control. First of all, in East China, rice is the staple food of three meals a day. Previous studies showed that higher consumption of rice was associated with an increased risk of diabetes $^{(36)}$. Second, pickled vegetables with high salt content have also been associated with higher risk of hypertension, an important risk for diabetes ${ }^{(37)}$. On the other hand, constituents of fruits and vegetables, including dietary fibre, antioxidants (e.g. vitamins C, E and folate) and $\mathrm{Mg}$, have been independently associated with reduced risk of diabetes ${ }^{(38)}$. Some constituents such as dietary fibre, antioxidants (e.g. vitamins C, E and folate) and magnesium found in fruits and vegetables have been shown to be associated with reduced risk of diabetes ${ }^{(38)}$. Jiang et al. have found that increasing dietary fibre intake may be an effective approach to reduce the level of HbA1c, improving glycaemic control in Chinese diabetic patients ${ }^{(39)}$. Moreover, an emerging body of evidence has demonstrated that antioxidants, such as vitamin $\mathrm{C}$ and other carotenoids compounds abundant in fruits and vegetable, are associated with decreased risk of obesity and hypertension, which are the established risk factor for $\mathrm{T}_{2} \mathrm{DM}^{(40,41)}$. Thus, these possibilities cannot be excluded in the analysis. 
In our study, higher adherence to the Western pattern was positively associated with poor glycaemic control. Similarly, in the study by Ruiz Martinez et al., the significant association between consuming a Western-style diet and HbA1c $\geq 7.0 \%$ was observed in Mexican population $^{(25)}$. The detrimental effect of this pattern on glycaemic control may be related to the unhealthy foods, e.g. red meat, processed and cooked meat, fast foods, snacks and chocolates. First, a previous study from our group in the sample found that higher consumption of red meat was positively associated with $\mathrm{T}_{2} \mathrm{DM}^{(16)}$. Alternatively, red meat is rich in haem Fe. Previous study has reported that body Fe overload may promote insulin resistance and increase the risk of $\mathrm{T}_{2} \mathrm{DM}^{(42)}$. Second, processed and cooked meats contain a lot of salt. The aforementioned findings suggest that high salt intake can increase the risk of diabetes ${ }^{(37)}$. Third, snack and chocolates with high glycaemic index could lead to an increase in weight gain, which can affect the metabolism of glucose and insulin sensitivity, thus increasing the level of blood glucose $^{(43)}$. Likewise, a high consumption of energy and energy $/ \mathrm{kg}$ of ideal weight has been shown to affect glycaemic control ${ }^{(44)}$. Finally, fast foods including in the Western pattern has been reported to be associated with an increased risk of diabetes ${ }^{(45)}$. A cross-sectional study in the United Arab Emirates found that fast-food consumption ( $>1$ time/week) had an OR of $1 \cdot 83$ (95\% CI 1.02, 3.3) for poor glycaemic control among diabetic patients ${ }^{(46)}$.

Higher adherence to the grains-vegetables pattern, composed mainly of whole grains, vegetables and beans, was positively associated with improving glycaemic control. Our results are in agreement with recent a study, which suggest that consuming a healthy pattern was associated with improved glycaemic control ${ }^{(25)}$. This favourable association of grains-vegetables pattern with improving glycaemic control may be attributed to this pattern's healthy constituents, for example whole grains, tuber, vegetable and beans. First, dietary fibre intake has been found to be associated with reduced risk of insulin resistance ${ }^{(38)}$. Second, most foods (such as whole grains, tubers, vegetables and bean) in this pattern have a low glycaemic load, which has been reported to be associated with a lower risk of $\mathrm{T} 2 \mathrm{DM}^{(46)}$. Third, our finding also indicated that participants who belonged to the fourth quartile of grainsvegetables pattern had a higher level of physical activity, compare with those in the lowest quartile. A recent meta-analysis of observational studies revealed that higher level of physical activity was associated with decreased risk of obesity, a major factor for diabetes ${ }^{(47)}$.

Notably, in the present work, we used the data set from the Hangzhou Nutrition and Health Survey, designed to investigate the relationship between diet and health consequences. Two previous studies by our groups have also examined the effects of dietary patterns on the risks of T2DM and pre-diabetes, respectively ${ }^{(16,29)}$. Shu et al..$^{(16)}$ identified three major dietary patterns in 1918 participants aged 45-59 years in Hangzhou city, Zhejiang province, in East China. Participants in the highest quartile of the grainsvegetables dietary pattern scores had a lower OR for T2DM $(\mathrm{OR}=0.72(95 \% \mathrm{CI} 0.596,0.952), P=0.04)$, compared with those in the lowest quartile. Subsequently, in the same study sample, Shen et al. ${ }^{(29)}$ found that Western pattern with a high content in meats, fast foods, snacks and chocolates was associated with higher risk of prediabetes. However, in contrast to the mentioned two studies ${ }^{(16,29)}$, this study possessed its unique features. In the selection process of study participants, we excluded those with diabetes or using insulin or oral hypoglycaemic agents, who may have changed their dietary behaviours by the time when the survey was carried out. The exclusion of study participants helps confirm our hypotheses in a healthy middle-aged population-based setting and minimises the chance of reverse causation. Moreover, our research used HbA1c $\geq 7.0$ as poor glycaemic control. In this study, these included participants have not been diagnosed with diabetes or impaired glucose tolerance. Therefore, our findings reinforced the importance of optimising dietary behaviours for a healthy middle-aged population.

\section{Strengths and limitations}

Our study has several strengths. First, to our knowledge, this is one of the few studies to assess the association between dietary patterns and glycaemic control in a middle-aged Chinese population. Our findings may be helpful to develop dietary recommendations for the early prevention of hyperglycaemia. Second, our study used the dietary pattern analysis, which considered the complexity of overall diet, facilitating dietary recommendations. Third, data on dietary intake were collected by face-to-face interview using a validated semi-quantitative FFQ. This FFQ enabled us to obtain more reliable information on dietary intake of participants in the past 12 months. Meanwhile, the validity and reproducibility of this FFQ has also been confirmed elsewhere ${ }^{(16)}$. Fourth, we have also adjusted for potential known confounders in multivariate regression models. Nevertheless, some limitations also exist in this study. First, the cross-sectional design of this study does not allow for a causal interpretation between dietary patterns and glycaemic control. Thus, future studies with longitudinal designs are needed to confirm the our findings. Second, though we adjusted for multiple confounding variables, the possibility of residual confounding remains in our study. Third, the dietary pattern analysis is somewhat subjective in decisions on the number of factors extracted, food group methods and the labelling of dietary patterns ${ }^{(17)}$. Finally, it is important to note that the generalizability of our findings may be limited because the sample of this study consisted of middle-aged adults, which were recruited from the municipal area of Hangzhou city, China. 


\section{Conclusions}

To conclude, the results of our study showed that the Western pattern was associated with an increased risk, whereas grains-vegetables pattern was associated with a decreased risk of poor glycaemic control. Our findings reinforced the importance of optimising dietary behaviours and could be helpful to develop nutrition guide for early IGT prevention in a healthy middle-aged population in China. Given the cross-sectional nature of this study, additional prospective studies are necessary to confirm these findings.

\section{Acknowledgements}

Acknowledgements: The authors thank all participants and staffs, in particular from Department of Nutrition, Zhejiang Hospital and Department of Endocrinology, the No.1 People's Hospital of Pinghu for their active cooperation and assistance. We also acknowledge the Medical Center for Physical Examination, Zhejiang Hospital for their important contributions to collection of data in the present study. Financial support: This study was supported by National Natural Science Foundation of China (grant number: 82004040), Natural Science Foundation of Zhejiang (grant number: LY17H030008), the joint construction of projects by provinces and the ministry of education (grant number: 2014PYA002), Traditional Chinese Medicine Research Project of Zhejiang (grant number: 2020ZB009) and Medical and Health research fund project of Zhejiang Province (grant number: 2017KY190). Conflicts of interest: The authors declare that there is no conflict of interest associated with this manuscript. Authorship: The authors' responsibilities were as follows: Z.Q. and Z.P.F. designed and conducted this research; S.L. and H.Y.Q. analysed data; Z.Q. and S.X.M. wrote this manuscript; Z.Q. had primary responsibility for the final content of the manuscript and all authors read and approved the final manuscript. Ethics of human subject participation: Written informed consent was obtained from all study participants prior to their participation in the survey. This study was conducted according to the guidelines laid down in the Declaration of Helsinki, and all procedures involving research study participants were approved by the Institutional Review Board of the Zhejiang Hospital (2014-KA-1).

\section{Supplementary material}

For supplementary material accompanying this paper visit https://doi.org/10.1017/S1368980021003931

\section{References}

1. International Diabetes Federation (2017) IDF Diabetes Atlas, 8th ed. Belgium: International Diabetes Federation.
2. Lozano R, Naghavi M, Foreman K et al. (2012) Global and regional mortality from 235 causes of death for 20 age groups in 1990 and 2010: a systematic analysis for the Global Burden of Disease Study 2010. Lancet 380, 2095-2128.

3. Xu Y, Wang L, He J et al. (2013) Prevalence and control of diabetes in Chinese adults. JAMA 310, 948-959.

4. Wang Z, Li X \& Chen M (2018) Socioeconomic factors and inequality in the prevalence and treatment of diabetes among middle-aged and elderly adults in China.J Diabetes Res $\mathbf{2 0 1 8}$, 1471808.

5. Zhang N, Du SM \& Ma GS (2017) Current lifestyle factors that increase risk of T2DM in China. Eur J Clin Nutr 71, 832-838.

6. Salas-Salvadó J, Bulló M, Estruch R et al. (2014) Prevention of diabetes with Mediterranean diets: a subgroup analysis of a randomized trial. Ann Intern Med 160, 1-10.

7. Zheng Y, Ley SH \& Hu FB (2018) Global aetiology and epidemiology of type 2 diabetes mellitus and its complications. Nat Rev Endocrinol 14, 88-98.

8. Cooper AJ, Sharp SJ, Lentjes MA et al. (2012) A prospective study of the association between quantity and variety of fruit and vegetable intake and incident type 2 diabetes. Diabetes Care 35, 1293-1300.

9. Geiker NRW, Larsen ML, Dyerberg J et al. (2018) Egg consumption, cardiovascular diseases and type 2 diabetes. Eur J Clin Nutr 72, 44-56.

10. Kurotani K, Nanri A, Goto A et al. (2013) Red meat consumption is associated with the risk of type 2 diabetes in men but not in women: a Japan public health center-based prospective study. Br J Nutr 110, 1910-1918.

11. Djoussé L, Petrone AB, Hickson DA et al. (2016) Egg consumption and risk of type 2 diabetes among African Americans: the Jackson Heart Study. Clin Nutr 35, 679-684.

12. Forouhi NG, Koulman A, Sharp SJ et al. (2014) Differences in the prospective association between individual plasma phospholipid saturated fatty acids and incident type 2 diabetes: the EPIC-InterAct case-cohort study. Lancet Diabetes Endocrinol 2, 810-818.

13. Soriguer F, Colomo N, Olveira G et al. (2013) White rice consumption and risk of type 2 diabetes. Clin Nutr 32, 481-484.

14. Mohan V, Radhika G, Sathya RM et al. (2009) Dietary carbohydrates, glycaemic load, food groups and newly detected type 2 diabetes among urban Asian Indian population in Chennai, India (Chennai urban rural epidemiology study 59). Br J Nutr 102, 1498-1506.

15. Ley SH, Hamdy O, Mohan V et al. (2014) Prevention and management of type 2 diabetes: dietary components and nutritional strategies. Lancet 383, 1999-2007.

16. Shu L, Shen XM, Li C et al. (2017) Dietary patterns are associated with type 2 diabetes mellitus among middle-aged adults in Zhejiang Province, China. Nutr J 16, 81.

17. Xu SS, Hua J, Huang YQ et al. (2020) Association between dietary patterns and chronic kidney disease in a middle-aged Chinese population. Public Health Nutr 23, 1058-1066.

18. Hu FB (2002) Dietary pattern analysis: a new direction in nutritional epidemiology. Curr Opin Lipidol 13, 3-9.

19. Huang L, Wang Z, Wang $\mathrm{H}$ et al. (2021) Nutrition transition and related health challenges over decades in China. Eur J Clin Nutr 75, 247-252.

20. Yuan YQ, Li F, Meng P et al. (2016) Gender difference on the association between dietary patterns and obesity in Chinese middle-aged and elderly populations. Nutrients $\mathbf{8}, 448$.

21. Zheng PF, Shu L, Zhang XY et al. (2016) Association between dietary patterns and the risk of hypertension among Chinese: a cross-sectional study. Nutrients $\mathbf{8}, 239$.

22. Zhu WL, Guan Y, Xu CZ et al. (2020) Influence of dietary patterns on type 2 diabetes mellitus in local residents aged 40 years and above in Songjiang district, Shanghai. Zhonghua Liu Xing Bing Xue Za Zhi 41, 508-513. 
23. Wei ZY, Liu JJ, Zhan XM et al. (2018) Dietary patterns and the risk of metabolic syndrome in Chinese adults: a population-based cross-sectional study. Public Health Nutr 21, 2409-2416.

24. Hakeem R, Ahmedani MY, Alvi SF et al. (2014) Dietary patterns and glycemic control and compliance to dietary advice among fasting patients with diabetes during Ramadan. Diabetes Care 37, e47-e48.

25. Martínez MLR, Gómez-Díaz RA, González ALV et al. (2020) Association between glycemic control and dietary patterns in patients with type 2 diabetes in a Mexican institute. Nutrition 78, 10901.

26. Jaacks LM, Crandell J, Mendez MA et al. (2015) Dietary patterns associated with HbA1c and LDL cholesterol among individuals with type 1 diabetes in China. $J$ Diabetes Complications 29, 343-349.

27. Yang Y (2005) Chinese Food Composition Table 2004. Beijing: Peking University Medical Press.

28. Wang CJ, Yang TF, Wang GS et al. (2018) Association between dietary patterns and depressive symptoms among middle-aged adults in China in 2016-2017. Psychiatry Res 260, 123-129.

29. Shen XM, Huang YQ, Zhang XY et al. (2020) Association between dietary patterns and prediabetes risk in a middleaged Chinese population. Nutr J 19, 77.

30. Yang CQ, Shu L, Wang S et al. (2015) Dietary patterns modulate the risk of non-alcoholic fatty liver disease in Chinese adults. Nutrients 7, 4778-4791.

31. Craig CL, Marshall AL, Sjöström M et al. (2003) International physical activity questionnaire: 12-country reliability and validity. Med Sci Sports Exerc 35, 1381-1395.

32. Zhu S (2021) Development of nutritional studies in China. Eur J Clin Nutr 75, 230-231.

33. Shu L, Zheng PF, Zhang XY et al. (2015) Association between dietary patterns and the indicators of obesity among Chinese: a cross-sectional study. Nutrients 7, 7995-8009.

34. Jaworowska A, Blackham T, Davies IG et al. (2013) Nutritional challenges and health implications of takeaway and fast food. Nutr Rev 71, 310-318.

35. Sarmento RA, Antonio JP, de Miranda IL et al. (2017) Eating patterns and health outcomes in patients with type 2 diabetes. J Endocr Soc 2, 42-52.
36. Hu EA, Pan A, Malik V et al. (2012) White rice consumption and risk of type 2 diabetes: meta-analysis and systematic review. BMJ 344, e1454.

37. Cryer MJ, Horani T \& DiPette DJ (2016) Diabetes and hypertension: a comparative review of current guidelines. J Clin Hypertens 18, 95-100.

38. Greenhill C (2020) Dietary factors in the risk of T2DM. Nat Rev Endocrinol 16, 537.

39. Jiang J, Qiu H, Zhao G et al. (2012) Dietary fiber intake is associated with HbA1c level among prevalent patients with type 2 diabetes in Pudong new area of Shanghai, China. PLOS One 7, e46552.

40. Garcia-Diaz DF, Lopez-Legarrea P, Quintero P et al. (2014) Vitamin $C$ in the treatment and/or prevention of obesity. J Nutr Sci Vitaminol 60, 367-379.

41. Nuñez-Cordoba JM, Alonso A, Beunza JJ et al. (2009) Role of vegetables and fruits in Mediterranean diets to prevent hypertension. Eur J Clin Nutr 63, 605-612.

42. Jiang R, Ma J, Ascherio A et al. (2004) Dietary iron intake and blood donations in relation to risk of type 2 diabetes in men: a prospective cohort study. Am J Clin Nutr 79, 70-75.

43. Al-Khudairy L, Stranges S, Kumar S et al. (2013) Dietary factors and type 2 diabetes in the Middle East: what is the evidence for an association? A systematic review. Nutrients $\mathbf{5}$, 3871-3897.

44. Evert AB, Boucher JL, Cypress M et al. (2014) Nutrition therapy recommendations for the management of adults with diabetes. Diabetes Care 37, Suppl. 1, S120-S143.

45. Pereira MA, Kartashov AI, Ebbeling CB et al. (2005) Fast-food habits, weight gain, and insulin resistance (the CARDIA study): 15-year prospective analysis. Lancet 365, 36-42.

46. Sugiyama M, Tang AC, Wakaki Y et al. (2003) Glycemic index of single and mixed meal foods among common Japanese foods with white rice as a reference food. Eur J Clin Nutr 57, 743-752.

47. Sharkey T, Whatnall MC, Hutchesson MJ et al. (2020) Effectiveness of gender-targeted $v$. gender-neutral interventions aimed at improving dietary intake, physical activity and/or overweight/obesity in young adults (aged 17-35 years): a systematic review and meta-analysis. Nutr J 19, 78. 